\title{
EARLY INTERVENTION AND IDENTIFICATION OF GAMBLING DISORDER: A SYSTEMATIC LITERATURE REVIEW OF STRATEGIES IMPLEMENTED BY GAMBLING OPERATORS
}

\author{
Kateřina Škařupová1, Tomáš Vlach², Viktor Mravčík ${ }^{2,3,4}$ \\ ${ }^{1}$ Institute for Research on Children, Youth and Family, Faculty of Social Studies, Masaryk University, Brno, Czech Republic \\ ${ }^{2}$ National Monitoring Centre for Drugs and Addiction, Office of the Government of the Czech Republic, Prague, Czech Republic \\ ${ }^{3}$ Department of Addictology, First Faculty of Medicine, Charles University and General University Hospital in Prague, Prague, Czech Republic \\ ${ }^{4}$ National Institute of Mental Health, Klecany, Czech Republic
}

\begin{abstract}
SUMMARY
Objective: Recent developments in online lotteries and betting and in digitalization of land-based gambling devices bring new opportunities to track behaviour of individual players and to identify and address developing problem in its initial stages. Early identification of gambling disorder allows for timely intervention and increases the likelihood of successful recovery and minimises harms. Our review aims to examine what on-site strategies are available in both online and offline gambling venues to early identify and address the developing gambling problem while also assessing their effectiveness and strength of the evidence.

Methods: We searched main academic databases and other internet resources and collected 67 peer-reviewed papers and grey literature documents that describe one or more such strategies.

Results: Available measures ranged from information provision, gambling behaviour surveillance and associated personalized interventions to setting limits and self-exclusion.

Conclusions: Although a number of methods how to address disordered gambling are available to gambling operators, there is still insufficient evidence about the validity and reliability of identification strategies and about effectiveness of the intervention methods.
\end{abstract}

Key words: gambling disorder, online gambling, early intervention and identification, prevention, operator-based approaches

Address for correspondence: K. Škařupová, Faculty of Social Studies, Masaryk University, Joštova 10, 60200 Brno, Czech Republic. E-mail: skarupovakat@gmail.com

https://doi.org/10.21101/cejph.a5849

\section{INTRODUCTION}

The Czech Republic has been recently undergoing a process of updating its gambling legislation as it was the case also in other European countries, partly to harmonize the laws with the EU legal acts, and partly to address a rather recent phenomenon of online gambling. Such major policy changes require solid evidence. Our analysis aims to provide summary of existing knowledge on early intervention and identification methods that can be implemented by the gambling operators, regulatory authorities and supervisory agencies in both online and offline gambling venues.

Extreme forms of compulsive gambling are among the conditions most difficult to treat, which is why prevention and harm reduction oriented approaches appear to be the most effective in terms of reducing individual and societal costs (1). Moreover, only a small proportion of those who suffer from gambling disorder seek formal treatment despite its availability and this treatment avoidance is often related to shame, denial or desire to handle the problem on their own. Early identification methods and in situ interventions represent an opportunity to overcome these obstacles, as brief treatments are often not perceived as treatment by individuals who access them (2).

Preventive measures may either aim to increase awareness through education and information campaigns or to limit access to gambling through regulated local availability, age restrictions and other regulatory measures (3). Within these categories, operators may inform about game principles and probabilities of wins, increase awareness of risks and symptoms of gambling disorder, educate and train staff and set up responsible gambling info points, while access may be restricted through self-imposed limits (of either time and money) and self-exclusion from the venue. Ariyabuddhiphongs (4) distinguishes between harm reduction model that focuses mainly on children and risk factors, and responsible gambling model, which represents a broader context involving various stakeholders including governments, gambling operators, and gamblers. Ariyabuddhiphongs (4) divides preventive measures according to their temporal sequence before, during and after development of gambling disorder. The before preventive measures involve, among others, correction of misconceptions about games and address attitudes toward gambling. The during 
measures involve structural changes to gambling machines and insertion of warning messages. The typical after measure represents voluntary self-exclusion.

Blaszczynski et al. (5) categorised interventions of operators to those facilitating awareness, facilitating control, and restricting access. Measures facilitating awareness focus on provision of information about principles of games, probability of win but also about own gambling behaviour (time, wages, loses) during gaming; also, on-site information centres in gambling venues belong into this category. Facilitating control consists mainly of self-commitment strategies in combination with cooling-off periods allowing gamblers to reconsider their decisions; limited access to (additional) financial resources may also reduce chasing losses. Measures restricting access are interventions limiting access to minors and under-aged to gambling and measures providing self-exclusion from gambling.

\section{MATERIALS AND METHODS}

We conducted a systematic literature review of early identification and intervention for problem/pathological gambling that are implemented by gambling operators in all environments and regardless the type of game. Three main academic databases, EbscoHOST (limited to Academic Search Complete, SocIndex with Full Text, PsycARTICLES, and PsycINFO), Scopus, and PubMed, were searched using Boolean expression for articles that contained at least one term for gambling (e.g. gambling or betting), level of problem (e.g. problematic, pathological, disorder, addiction, or at-risk), intervention (e.g. assessment, identification, monitoring, care, or response), and environment (e.g. early, on-site, venue, online, or algorithm). The search was conducted between September and November 2015. Only papers published in English, in 2000 or later, and containing systematic description of either in situ identification of problem gamblers or in situ intervention for problem gambling, or, alternatively, a comprehensive system of such measures were selected for analysis. By systematic description we mean any description of a study, guidelines or intervention, therefore, texts containing only mention of such interventions (such as newspaper articles, commentaries, editorials, epidemiological descriptions of gambling populations, etc.) were not included. In situ denotes any measure that is implemented directly within the gambling environment - casino, gambling venue, slot-machine, or online gambling website; interventions that were implemented elsewhere (e.g. help-lines), by other providers than the gambling operators (e.g. treatment providers), and structured long-term and/or repeated interventions (e.g. therapy sessions) were not included. No additional exclusion criteria were specified in terms of quality of scientific reporting and/or strength of the evidence as we intended to provide a complete overview of all available strategies while also assessing their effectiveness and the cogency of arguments.

The inclusion criteria were tested by three independent researchers on a subsample of 50 entries; the results were compared and discussed. The final sampling procedure comprised the following steps: first, the search string was applied in each database separately, the titles were scanned and potentially relevant papers were downloaded to a citation manager, the results from the three databases were merged and duplicates were removed.
Second, on the basis of abstract, full-texts of relevant papers were downloaded where available. Third, additional papers known to authors beforehand and also relevant papers referenced by the ones previously identified were added to the final sample (Fig. 1). Here we present a narrative summary of the evidence.

\section{RESULTS}

The interventions described in the 67 selected papers were classified into four major categories: provision of information (pop-up messages and static information provision); gambling behaviour surveillance and related interventions (land-based environment surveillance, online gambling data, and personalised feed-back and targeted pop-up messages); setting limits - pre-commitment; exclusion and self-exclusion.

\section{Provision of Information}

Provision of information usually aims to change knowledge and attitudes of gamers, to correct their erroneous beliefs about gaming principles, and subsequently, to change their behaviour. These information services may take various forms, from simple leaflets campaigns to pop-up messages, to complex educational centres build within the gambling venues. Static information is often a minimum requirement of the national or local guidelines and policy documents obliging gambling operators and venues, for instance, to visibly display information about responsible gambling principles, risks, and help resources, information on probability of wins and game rules and principles (6). Available research shows that this type of intervention serves more as a demonstration of a good will of gambling operators rather than effective prevention strategy as it often remains overlooked by gamers who do not pay attention to such messages $(7,8)$.

To increase effectiveness of information provision, gaming sessions should be paused or interrupted to draw the attention of gamers to the message. To increase remembering, the message content should involve emotionally strong statement or provoke self-appraisal, and should be attributed to a credible source ( 9 , 10). Dynamic messages that appeared in the middle of the screen are more remembered and considered effective than messages displayed either on top, or on the bottom of the screen (11). Messages attributed to a credible source (e.g. public health authority) are trusted more when compared to messages from the gambling website operators (8). Self-evaluating messages and messages referring to amount of money spent are more remembered than those that contain simple information statement (12). People who were shown fearful statement (e.g. warning from the risk of dysfunctional relationships or about the risk of suicide) declared higher willingness to change their gambling behaviour and could recall the content of the message more often (8). In terms of information and knowledge delivery, these pop-up messages appear to be similarly effective as short (up to 9 minutes) educational video spots, while also being more applicable in the real environment $(13,14)$.

Although remembered, recalled, and considered useful, such messages often fail to change the actual gambling behaviour (11). For instance, Ariyabuddhiphongs (4) concludes that despite forced break in gaming session and increased awareness of game mechanics and probabilities of win, the pop-up messages did not lead to 


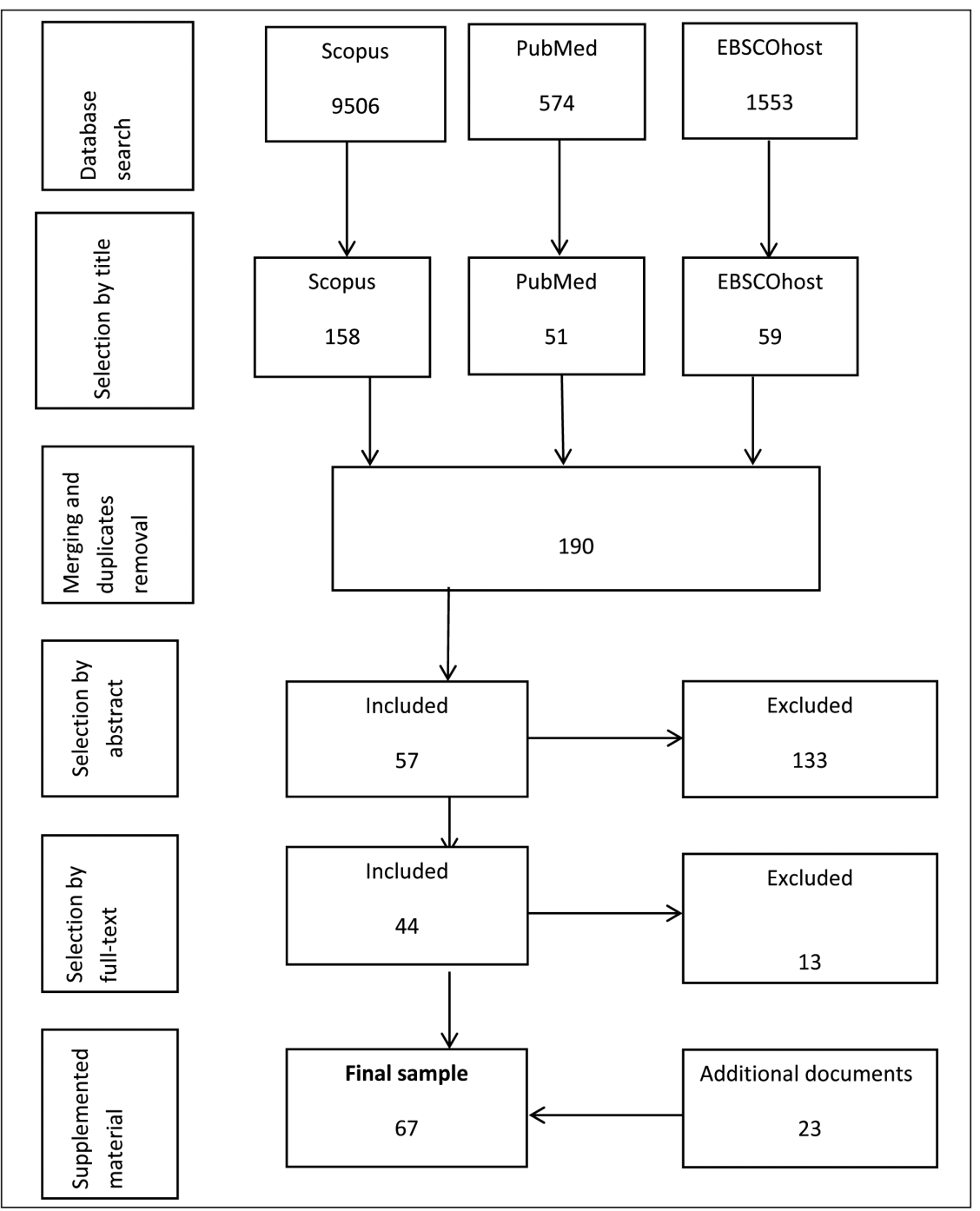

Fig. 1. Sampling procedure scheme.

reduction of the gambling activity. Similarly, gamblers exposed to educational pop-up messages during play did not differ in number of game cycles compared to control group that did not see any messages while their game was interrupted for the same period of time (15). Any effect of the information and education provided is probably moderated by the level of pathology: gamblers at risk may be more prone to change their attitudes in the light of the provided information than those with severe gambling disorder (16). This moderation may be related to pre-existing comorbidity of impulsecontrol disorders and associated gambling disinhibition (5).

\section{Early Identification Methods in Land-based Envi- ronment}

Methods of early identification of developing gambling problem in situ usually involve observation of behavioural indicators by the venue staff and, eventually, face-to-face intervention. Known symptoms of pathological gambling include physiological symptoms (e.g. increased sweating, nausea, headaches, dry eyes), emotional reactions to play (e.g. depressive symptoms, irritability, aggression, complaining, or crying), gambling patterns and habits (e.g. gambles more than once a week, in long sessions of over two hours, increases bets, does not take breaks, increases frequency and the time and amount spent on gambling), financial transactions (e.g. attempts to sell personal belongings or borrow money on site, frequent ATM withdrawals, often requires change), social activity (e.g. avoids company, comes alone and/ or secretly, neglected appearance), and anti-social behaviours such as licit and illicit drug use, or neglecting family and work (17-23). Despite extensive symptomatology potentially allowing for identification of problematic gamblers in the gambling venue, proactive approach of the venue staff appears to be difficult and its implementation in practice is rather rare. Some of the indicators, such as amount of money lost in game or time spent gambling are often considered individual and/or relative and therefore they are seldom used by the staff (24). The personnel often report lack of relevant advisory and therapeutic skills, lack of confidence when approaching a gambler that did not ask for help, and also the lack of clarity in terms of competences and responsibilities (5). Other obstacles are related to organization of work in venues due to changing of shifts, short-term and inconsistent observations (25). Education of staff may increase their confidence in recognizing and approaching problematic gamblers that did not ask for help themselves, however, their ability, motivation, and willingness to utilize these skills and knowledge in practice deteriorates in time favouring repeated trainings over short-term or one-session 
programmes (26). Allcock $(22,23)$ argues that venue personnel should be able to recognize emerging gambling problem, however, they should only refer the individual to a specialized service rather than intervene and/or attempt to provide therapeutic help for which they are not sufficiently qualified.

\section{Online Gambling Behaviour Surveillance}

Compared to observations in the land-based venues, online gambling systems provide rather precise and structured data and some authors have great expectations from data-mining techniques and algorithms in terms of early identification of problematic gamblers (27). This type of data might help to overcome obstacles associated with judgement of the venue staff that may overlook some symptoms, pay intermittent attention, or be under-trained (25). Indicators of online problematic gambling patterns mirror offline symptoms and include between-session indicators (e.g. frequency of gambling, length of individual gambling session, money spent, wins and losses, chasing losses) and within-session indicators (e.g. topping-up, credit cards changes, variability of bets, gambling patterns, volatility) $(28,29)$. Data from the first month of the online activity usually suffice to predict gamblers who will place higher bets, experience greater losses, and develop gambling disorder in the future (30-32). Automation may be applied also on textual data from the customer help lines and service desks with similar results compared to analysis of the gambling behaviours attempts to increase betting limits, complains, criminal activities and threats, problematic family and social situation, health and financial problems indicate potential need for intervention $(33,34)$.

Limits of these approaches are associated to invisible end-users who may share their online accounts with others or use numerous accounts in more operators $(35,36)$. Self-imposed limits, selfexclusions, account terminations, and reasons behind are often the only available indicators of emerging problem which reduces validity of such studies (37). Complex estimation methods also appear to improve results compared to simple measures (such as limit for loss or length of one gambling session) only minimally. For instance, Excell et al. (38) developed complex data-driven method to identify pathological gamblers using the Problem Gambling Severity Index (PGSI) as a gold standard and reached sensitivity of only $25 \%$ while keeping specificity around $90 \%$.

\section{Targeted Pop-up Messages and Personalised Feedback}

Data on individual gambling behaviours may be utilized for early interventions in the form of personalised pop-up messages and feedback. Auer and Griffiths (39) explored efficiency of pop-up messages with the following content: "You have now played 1,000 slot games. Do you want to continue?" In the sample of 400,000 game sessions, around $1 \%$ reached the limit of 1,000 games that correspond to approximately one hour of play. Nine times more sessions were terminated after the implementation of the pop-up message ( 5 compared to 45 sessions). More complex feedback including also time of play, summary of wins and losses provided in graphical and textual forms significantly reduced financial losses and gambling time and improved ability to self-evaluate and regulate gambling behaviour (39). When intervention and feedback are individually tailored on the basis of personal data, they are more likely positively received and trusted compared to general statements (40). Online applications utilizing analysis of individual data, assessing the level of risk, and offering a whole range of responses and sources of help, including on-site measures such as setting limits and self-exclusions, are being developed (41).

\section{Limits, Pre-commitments and Self-exclusion}

Players may restrict financial flows, time spent in gambling, and access to both online and offline gambling venues. Especially online gambling sites provide a broad scale of pre-commitment possibilities. Financial limits may involve deposits (maximum amount of money available for gambling), assets (maximum amount money active in one gambling session), losses and bets (maximum value of loss and maximum value of a bet within one gambling opportunity). Limits may be either voluntary or compulsory, and either fixed (the same limits apply for all gambling sessions within one account) or variable (42). Compulsory limits are, however, often pre-set to a default value that is relatively high and rarely reached by majority of gamblers, and as such, they do not represent an effective prevention tool (43). Voluntary self-imposed limits, especially when combined with a certain time-period to cool-off (limits can be increased, but the change becomes active after some time, typically 72 hours), have been shown to have a positive impact on time spent in gambling and on the amount of money lost (39). Voluntary limits appear to be used mainly by the players who already experienced loss of control over their gambling and for whom the limits also proved to have the strongest effect on lost money and time (44). A simulation study examined limits imposed on wins and concluded that they may also lead to a significant reduction of losses (45). Overall, self-imposed limits and pre-commitment strategies show moderate effect, however, only when they are compulsory, irreversible, and applicable through all gambling opportunities within a country (3).

Self-exclusion, considered the utmost protective measure, is often utilised by the most problematic gamblers with developed gambling disorder. The self-exclusion programme in land-based gambling venues usually consists of three components: voluntary self-exclusion; expulsion from the premises and/or fine after violation of the self-exclusion; forfeiture of any win achieved during selfexclusion. Some programmes add fourth component of treatment referral (4). Even though the majority of self-excluded players break the commitment at some point, it still remains effective in terms of positive impact on financial, social and psychological situation of gamblers $(4,5)$. Gainsbury $(46)$ in her review identified number of positive effects of self-exclusion including decrease of money used for one bet, stabilisation of financial situation, less frequent play and less time spent in game, reduction of symptoms of gambling disorder, less psychological distress (lower depressions and anxiety), increased sense of control over one's gambling and overall.

Limits of the self-exclusion programmes are related mainly to the structural obstacles - the procedure is often complicated and involves heavy paper work, it is valid only for one casino/ operator, it is not consistently reinforced by the security staff, nor promoted among gamblers, and is associated with stigma (46-49). Adherence to the programme also deteriorates with time and decreasing motivation, however, combination with parallel intervention did not increase the positive effect of self-exclusion $(50,51)$. Technological advancements may solve some of these problems through possibility of multi-operator self-exclusion (5). 


\section{DISCUSSION}

A number of tools and measures to prevent, identify and minimize negative impact of problematic gambling are available to gambling operators. A broad range of behavioural clues and observable symptoms in both online and land-based gambling environments were identified. Nevertheless, there is still lack of systematic evidence and scientific consensus over the critical values that would allow for reliable distinction between problematic and non-problematic gamblers. For instance, some of the indicators are derived from the diagnostic criteria of pathological gambling/gambling disorder which require certain insight into the background of the individuals which is not always possible on site. Similarly, automatic detection in online gambling systems seems promising, however, it would require more research, more precision in terms of gold standard, and centralised databases that would allow to track one user across more platforms, operators, and betting systems (20). Methods of early identification of individuals at risk of developing gambling disorder will require more evidence on various gambling patterns and courses of the disorder that often involves binge and episodic gambling that may be easily missed using standard measures $(52,53)$.

Interventions vary from unobtrusive (and the least effective) static information provision in the online or land-based gambling venues and methods involving feedback and personal interaction with a staff member to limited or banned access. Differences exist also in terms of participation of the gambler and level of his/her self-reflection and problem-awareness. Gambling operators and staff, on the other hand, are expected to show high responsibility, awareness, knowledge, and confidence in terms of recognizing the symptoms of developing problem and acting towards the minimization of harms. Research shows, however, that these expectations are rarely met and the operators often lack desired qualifications and their activities are limited whenever they might put their revenue at risk (54).

Effects of the early interventions appear to be moderated by the severity of the gambling disorder with more severe cases being less susceptible to change (10). The nature of the problem that involves loss of control over one's behaviour offers an explanation of the moderating effect - the behaviours associated with gambling disorder, such as chasing losses and inability to stop, often result from greater impulsivity, are poorly thought out and premature (2). The moderation effect will be most pronounced for the least obtrusive intervention methods, such as information provision, while self-exclusion and self-imposed limits may compensate for deficits in inhibition, time management, working memory, and planning in the most problematic gamblers (2).

Although information provision seems rather ineffective as a prevention and harm reduction measure, especially when no game interruption and self-assessment are involved, campaigns and training towards general public and disordered gamblers are important to increase awareness about the aetiology, risks and consequences of gambling disorder. Greater understanding to pathological gambling may help to reduce stigma associated with the disorder and, subsequently, increase the treatment uptake (55). The demand for treatment, generally low among problem gamblers, might be also elevated through better cooperation and link between the operators and treatment providers. For instance, information and referral to conventional treatment and/or brief intervention services should be available on site and provided in a sensitive, stigma-reducing manner (48).

\section{CONCLUSIONS}

Resources identified in our review do not provide much certainty about the effectivity of the individual methods of early identification and intervention implemented by the gambling operators. Some evidence comes from the isolated studies, studies conducted in laboratory settings, others have strong limits in terms of progress indicators that rely on self-assessment of respondents and do not measure actual change of behaviour (5). Recent review of available approaches concluded that gambling prevention and on-site interventions have been in general haphazard initiatives, and similar lack of systematic approach applies also for the evaluation studies in the area (56). With the exception of longterm measures such as self-imposed limits and self-exclusion, there was a lack of focus on sustainability and persistence of the effects. Analyses based on online gambling databases may be precise, but often fail to capture the problematic gambler across multiple environments and, therefore, may not be a good indicator at all (20). To overcome these obstacles, more research is needed on different patterns associated with specific game types, taking account of variability in disorder progression and involving real gamblers in real life settings rather than laboratory experiments and computer simulations (2). The contradictory or in-conclusive evidence should not prevent national regulators and policy makers from making necessary protective measures and addressing the risks associated with gambling as a public health concern (57).

\section{Acknowledgements}

The review was prepared within the project CZ.1.04/4.1.00/B6.00030 coordinated by the Ministry of Finance of the Czech Republic and co-financed by the European Social Fund. The authors acknowledge institutional support programme Progress No. Q06/LF1 and the Project No. LO1611, with financial support from the Ministry of Education, Youth, and Sports of the Czech Republic as part of the NPU I programme.

\section{Conflict of Interests}

None declared

\section{REFERENCES}

1. Meyer G, Hayer T, Griffiths M, editors. Problem gambling in Europe: challenges, prevention, and interventions. New York: Springer; 2009.

2. Hodgins DC, Stea JN, Grant JE. Gambling disorders. Lancet. 2011;378(9806):1874-84.

3. Williams RJ, West BL, Simpson RI. Prevention of problem and pathological gambling: a comprehensive review of the evidence. Guelph: Ontario Problem Gambling Research Centre; 2008.

4. Ariyabuddhiphongs V. Problem gambling prevention: before, during, and after measures. Int J Ment Health Addict. 2013;11(5):568-82.

5. Blaszczynski A, Parke A, Parke J, Rigbye J. Operator-based approaches to harm minimisation in gambling: summary, review and future directions. London: The Responsible Gambling Trust; 2014.

6. Breen H, Buultjens J, Hing N. Perceived efficacy of responsible gambling strategies in Queensland hotels, casinos and licensed clubs [Internet]. Lismore: Southern Cross University; 2003 [cited 2019 Dec 5]. Available from: https://epubs.scu.edu.au/cgi/viewcontent. cgi? article $=1277 \&$ context $=$ comm_pubs. 
7. Monaghan S, Blaszczynski A, Nower L. Do warning signs on electronic gaming machines influence irrational cognitions? Psychol Rep. 2009;105(1):173-87.

8. Munoz Y, Chebat JC, Suissa JA. Using fear appeals in warning labels to promote responsible gambling among VLT players: the key role of depth of information processing. J Gambl Stud. 2010 Dec;26(4):593-609.

9. Monaghan S. Responsible gambling strategies for Internet gambling: the theoretical and empirical base of using pop-up messages to encourage self-awareness. Comput Hum Behav. 2009;25(1):202-7.

10. Monaghan S, Blaszczynski A. Electronic gaming machine warning messages: information versus self-evaluation. J Psychol. 2010;144(1):83-96.

11. Gainsbury S, Aro D, Ball D, Tobar C, Russell A. Determining optimal placement for pop-up messages: evaluation of a live trial of dynamic warning messages for electronic gaming machines. Int Gambl Stud. 2015;15(1):141-58

12. Gainsbury SM, Aro D, Ball D, Tobar C, Russell A. Optimal content for warning messages to enhance consumer decision making and reduce problem gambling. J Bus Res. 2015;68(10):2093-101.

13. Wohl MJA, Santesso DL, Harrigan K. Reducing erroneous cognition and the frequency of exceeding limits among slots players: A short (3-minute) educational animation facilitates responsible gambling. Int J Ment Health Addict. 2013;11(4):409-23.

14. Wohl MJA, Gainsbury S, Stewart MJ, Sztainert T. Facilitating responsible gambling: the relative effectiveness of education-based animation and monetary limit setting pop-up messages among electronic gaming machine players. J Gambl Stud. 2013;29(4):703-17.

15. Cloutier M, Ladouceur R, Sévigny S. Responsible gambling tools: popup messages and pauses on video lottery terminals. J Psychol. 2006 Sep;140(5):434-8.

16. Monaghan S, Blaszczynski A. Impact of mode of display and message content of responsible gambling signs for electronic gaming machines on regular gamblers. J Gambl Stud. 2010;26(1):67-88.

17. Schellinck T, Schrans T. Identifying problem gamblers at the gambling venue: finding combinations of high confidence indicators. Gambl Res. 2004 May; 16(1):8-24.

18. Haefeli J, Lischer S, Schwarz J. Early detection items and responsible gambling features for online gambling. Int Gambl Stud. 2011 Dec;11(3):273-88

19. Delfabbro PH, Osborn A, McMillen J, Neville M, Skelt L. The identification of problem gamblers within gaming venues: final report. Melbourne: Victorian Department of Justice; 2007.

20. Griffiths M, Whitty M. Online behavioural tracking in Internet gambling research: ethical and methodological issues. Int J Internet Res Ethics. 2010;3(12):104-17.

21. Xuan Z, Shaffer H. How do gamblers end gambling: longitudinal analysis of internet gambling behaviors prior to account closure due to gambling related problems. J Gambl Stud. 2009;25(2):239-52.

22. Blaszczynski A. Problem gambling behaviours: what can be observed in venues and how should staff respond? In: Allcock C, editor. Current issues related to identifying the problem gambler in the gambling venue. Melbourne: Australasian Gaming Council; 2002.

23. Allcock C, editor. Current issues related to identifying the problem gambler in the gambling venue. Melbourne: Australian Gaming Council; 2002.

24. O'Mahony B, Ohtsuka K. Responsible gambling: sympathy, empathy or telepathy? J Bus Res. 2015;68(10):2132-9.

25. Delfabbro P, Borgas M, King D. Venue staff knowledge of their patrons' gambling and problem gambling. J Gambl Stud. 2012;28(2):155-69.

26. Giroux I, Boutin C, Ladouceur R, Lachance S, Dufour M. Awareness training program on responsible gambling for casino employees. Int J Ment Health Addict. 2008;6(4):594-601.

27. Griffiths M. Internet gambling: issues, concerns, and recommendations. Cyberpsychol Behav. 2003 Dec;6(6):557-68.

28. Quilty LC, Avila Murati D, Bagby RM. Identifying indicators of harmful and problem gambling in a Canadian sample through receiver operating characteristic analysis. Psychol Addict Behav. 2014;28(1):229-37.

29. Wardle H, Parke J, Excell D. Machines research programme: Report 1 Theoretical markers of harm for machine play in a bookmaker's: a rapid scoping review. Responsible Gambling Trust; 2014.

30. Dragicevic S, Tsogas G, Kudic A. Analysis of casino online gambling data in relation to behavioural risk markers for high-risk gambling and player protection. Int Gambl Stud. 2011;11(3):377-91.

31. Braverman J, Shaffer HJ. How do gamblers start gambling: identifying behavioural markers for high-risk internet gambling. Eur J Public Health. 2012;22(2):273-8.

32. Braverman J, LaPlante DA, Nelson SE, Shaffer HJ. Using cross-game behavioral markers for early identification of high-risk internet gamblers. Psychol Addict Behav. 2013;27(3):868-77.
33. Haefeli J, Lischer S, Schwarz J. Early detection items and responsible gambling features for online gambling. Int Gambl Stud. 2011;11(3):273-88.

34. Gray HM, LaPlante DA, Shaffer HJ. Behavioral characteristics of Internet gamblers who trigger corporate responsible gambling interventions. Psychol Addict Behav. 2012;26(3):527-35.

35. Auer M, Schneeberger A, Griffiths MD. Theoretical loss and gambling intensity: A simulation study. Gaming Law Rev Econ. 2012;16(5):269-73.

36. Auer M, Griffiths MD. Voluntary limit setting and player choice in most intense online gamblers: an empirical study of gambling behaviour. J Gambl Stud. 2013;29(4):647-60.

37. LaBrie R, Shaffer HJ. Identifying behavioral markers of disordered Internet sports gambling. Addict Res Theory. 2011;19(1):56-65.

38. Excell D, Bobashev G, Gonzalez-Ordonez D, Wardle H, Whitehead T, Morris RJ, et al. Report 3: Predicting problem gamblers: analysis of industry data. Gambling machines research program. Responsible Gambling Trust; 2014.

39. Auer MM, Griffiths MD. The use of personalized behavioral feedback for online gamblers: an empirical study. Front Psychol. 2015;6:1406.

40. Cunningham JA, Hodgins DC, Toneatto T, Murphy M. A randomized controlled trial of a personalized feedback intervention for problem gamblers. PLoS ONE. 2012;7(2):e31586. doi: 10.1371/journal.pone.0031586.

41. Griffiths MD, Wood RTA, Parke J. Social responsibility tools in online gambling: a survey of attitudes and behavior among internet gamblers. Cyberpsychol Behav. 2009 Aug;12(4):413-21.

42. Wood RTA, Griffiths MD. Social responsibility in online gambling: voluntary limit setting. World Online Gambl Law Rep. 2010;9(11):10-1.

43. McMillen J, Pitt S. Review of the ACT Government's harm minimisation measures. Canberra: Australian National University, Centre for Gambling Research; 2005.

44. Nelson SE, LaPlante DA, Peller AJ, Schumann A, LaBrie RA, Shaffer HJ. Real limits in the virtual world: self-limiting behavior of Internet gamblers. J Gambl Stud. 2008;24(4):463-77.

45. Walker DM, Litvin SW, Sobel RS, St-Pierre RA. Setting win limits: an alternative approach to 'responsible gambling'? J Gambl Stud. 2015 Sep;31(3):965-86.

46. Gainsbury SM. Review of self-exclusion from gambling venues as an intervention for problem gambling. J Gambl Stud. 2014;30(2):229-51.

47. Sasso WV, Kalajdzic J. Do Ontario and its gaming venues owe a duty of care to problem gamblers? Gaming Law Rev. 2006;10(6):552-70.

48. Hing N, Tolchard B, Nuske E, Holdsworth L, Tiyce M. A process evaluation of a self-exclusion program: a qualitative investigation from the perspective of excluders and non-excluders. Int J Ment Health Addict. 2014 Aug; 12(4):509-23.

49. Hing N, Nuske E. Responding to problem gamblers in the venue: role conflict, role ambiguity, and challenges for hospitality staff. J Hum Resour Hosp Tour. 2012;11(2):146-64.

50. Ladouceur R, Sylvain C, Gosselin P. Self-exclusion program: a longitudinal evaluation study. J Gambl Stud. 2007;23(1):85-94.

51. Hing N, Russell A, Tolchard B, Nuske E. Are there distinctive outcomes from self-exclusion? An exploratory study comparing gamblers who have self-excluded, received counselling, or both. Int J Ment Health Addict. 2015;13(4):481-96.

52. Wohl MJA, Lyon M, Donnelly CL, Young MM, Matheson K, Anisman H. Episodic cessation of gambling: a numerically aided phenomenological assessment of why gamblers stop playing in a given session. Int Gambl Stud. 2008;8(3):249-63.

53. Nower L, Blaszczynski A. Binge gambling: a neglected concept. Int Gambl Stud. 2003;3(1):23-35.

54. Khazaal Y, Chatton A, Bouvard A, Khiari H, Achab S, Zullino D. Internet poker websites and pathological gambling prevention policy. J Gambl Stud. 2013;29(1):51-9.

55. Rüsch N, Corrigan PW, Wassel A, Michaels P, Larson JE, Olschewski M, et al. Self-stigma, group identification, perceived legitimacy of discrimination and mental health service use. Brit J Psychiatry. 2009 Dec;195(6):551-2.

56. Ladouceur R, Shaffer P, Blaszczynski A, Shaffer HJ. Responsible gambling: a synthesis of the empirical evidence. Addict Res Theory. 2017; 25(3):22535 .

57. Hancock L. Smith, G. Critiquing the Reno Model I-IV international influence on regulators and governments (2004-2015) - the distorted reality of 'responsible gambling'. Int J Ment Health Addict. 2017;15(6):1151-76.

Received June 2, 2019

Accepted in revised form December 5, 2019 